\title{
Effect of fiber-premixed indirect resin composite substructure on fracture resistance of MOD composite inlays adhered with two different adhesive resin cements
}

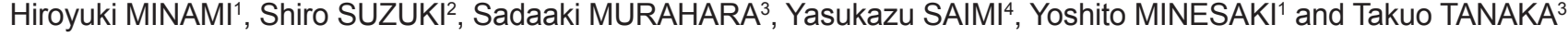 \\ ${ }^{1}$ Fixed Prosthetic Clinic, Kagoshima University Medical and Dental Hospital, 8-35-1 Sakuragaoka, Kagoshima 890-8544, Japan \\ ${ }^{2}$ Department of Prosthodontics, University of Alabama at Birmingham, School of Dentistry, $19197^{\text {th }}$ Ave. South, Birmingham, AL 35294-0007, USA \\ ${ }^{3}$ Department of Fixed Prosthetic Dentistry, Kagoshima University Graduate School of Medical and Dental Sciences, 8-35-1 Sakuragaoka, Kagoshima \\ 890-8544, Japan \\ ${ }^{4}$ Research Department, Sun Medical Co. Ltd., 571-2 Furutaka-cho, Moriyama, Shiga 524-0044, Japan \\ Corresponding author, Hiroyuki MINAMI; E-mail: minami@dentb.hal.kagoshima-u.ac.jp
}

This study evaluated the effect of a fiber-premixed indirect resin composite (FMC) substructure on the fracture resistance of mesialocclusal-distal (MOD) indirect composite restorations adhered to extracted human upper premolars. The teeth received a standardized MOD cavity preparation, and indirect composite inlays were fabricated with or without using the FMC. Inlays were cemented into the cavity preparations using either Super-Bond C\&B or Panavia F2.0. A total of 28 specimens, namely seven specimens for four groups, were thus fabricated. Failure load and failure energy were determined after thermocycling $\left(4-60^{\circ} \mathrm{C}\right.$ for $\left.5,000 \mathrm{cycles}\right)$. In terms of failure load, no significant differences were found among the four groups. In terms of failure energy, FMC substructure exerted a significantly favorable effect on Super-Bond C\&B-bonded group but a negative one on Panavia F2.0-bonded group. In conclusion, the failure energy of the group using FMC substructure and which was adhered using Super-Bond C\&B was significantly higher than the other groups.

Keywords: Fiber-premixed indirect resin composite, Indirect resin composite inlay, Fracture resistance

Received Nov 28, 2008: Accepted Mar 24, 2009

\section{INTRODUCTION}

Indirect composite restorations have been widely used for inlays, onlays, crowns, and fixed partial dentures because of improvements in physical and mechanical properties $^{1)}$, wear resistance ${ }^{2}$, and color stability ${ }^{3)}$. In a closely related manner, advances in adhesion technique to abutment teeth ${ }^{4}$ also helped spur the popularity of indirect composite restorations.

With composite restorations, clinicians often encounter crack formation or bulk fracture due to the brittleness of composite materials. To prevent bulk fracture, reinforcement techniques with glass and/or polymer fibers were developed. In terms of clinical application, some indirect composite restorative systems using pre-impregnated fibers have been introduced. Some in vitro studies have reported that fiber incorporation increased the fracture resistance of indirect resin composite crowns $^{5,6}$ and bridges ${ }^{7,8}$, whereas others ${ }^{9,10)}$ concluded that composite materials did not benefit from fiber reinforcement for crowns.

Recently, a novel fiber-premixed indirect resin composite (FMC) was developed ${ }^{11)}$ as a core material for indirect composite restorations, which does not necessitate further fiber incorporation. Satisfactory interfacial bonding between the FMC and indirect composite, and improved toughness and impact resistance of the indirect resin composite have been reported ${ }^{12)}$. Although most reinforced composite systems are applicable for fabrication of crowns ${ }^{5,6,9,10)}$ and bridges ${ }^{7,8)}$, the FMC can be used only for single tooth restorations as a core material in crowns, onlays, and inlays.

The hypothesis to be tested in this study was that the use of FMC would improve the fracture resistance of indirect composite restorations. In line with this hypothesis, the purpose of this in vitro study was to evaluate the effect of this FMC as a substructure on the fracture resistance of class II mesial-occlusal-distal (MOD) indirect composite restorations adhered to extracted human upper premolars using two different adhesive resin cements.

\section{MATERIALS AND METHODS}

Materials used

Materials used in this study are presented in Table 1. Fiber-premixed indirect resin composite (Jacket Opaque JMO, A3 opaque shade: FMC, Sun Medical Co. Ltd., Moriyama, Japan) and a microfilled indirect resin composite (Meta Color Prime Art Body Paste A3-B, A3 body shade: IC, Sun Medical Co. Ltd.) were used as inlay materials. Panavia F2.0 (PV, Kuraray Medical Inc., Tokyo, Japan) and Super-Bond C\&B (SB, Sun Medical Co. Ltd.) were used as luting materials.

The composition of FMC has been reported by a dental manufacturer and detailed in a previous study ${ }^{12)}$. It contained UDPAC as comonomer at $41.93 \mathrm{wt} \%$ and TEGDMA as matrix resin at $27.82 \mathrm{wt} \%$, as well as silanized milled glass fiber at $24.84 \mathrm{wt} \%$ and colloidal silica as filler at $4.97 \mathrm{wt} \%$. The organic components of FMC were dissolved in acetone for 60 minutes, and the 
residual glass fibers were air-dried and gold-coated using an ion-sputtering device (Fine Coat Sputter, JCF1100, JEOL, Tokyo, Japan). When observed under a scanning electron microscope (SEM) (JSM-1100, JEOL), it was shown that FMC contained silanized milled glass fibers with a diameter of $11 \mu \mathrm{m}$ and an average length of $150 \mu \mathrm{m}$ (Fig. 1).

\section{MOD cavity preparation}

Twenty-eight maxillary premolars were collected immediately after extraction and stored in isotonic sodium chloride solution at $4^{\circ} \mathrm{C}$ before being used in this study. They were nearly identical in size (buccalpalatal width: $9.52 \pm 0.29 \mathrm{~mm}$, mesial-distal width: $7.59 \pm 0.26 \mathrm{~mm}$, coronal height: $8.89 \pm 0.32 \mathrm{~mm}$ ), and all of them were intact and caries-free. The apex of each root was sealed with adhesive resin cement (SuperBond C\&B) without removal of pulp tissue, and then the teeth were mounted into stainless molds perpendicular to their long axis using an autopolymerizing resin. A standardized class II mesialocclusal-distal (MOD) cavity with convergent walls was prepared in all teeth (Fig. 2) with rotating diamond burs (SF206CR and SF103CR, Shofu Inc., Kyoto, Japan) under copious water spray. A high-speed handpiece combined with a dental surveyor (Ney Surveyor, JM Ney Co., CT, USA) was used to achieve standardized cavity preparations.

Impressions of the preparations were made using a polyvinylsiloxane impression material (Exafine, GC Corp., Tokyo, Japan) with a two-step impression

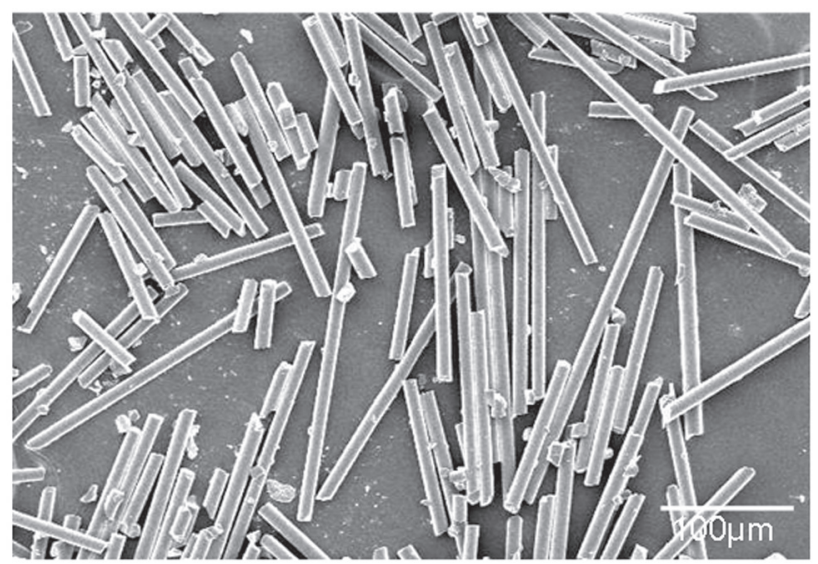

Fig. 1 SEM micrograph of the milled glass fibers contained in FMC.

Table 1 Materials used in this study

\begin{tabular}{|c|c|c|c|c|c|}
\hline Material & Product name & Abbreviation & Component & Manufacturer & Lot No. \\
\hline \multirow{2}{*}{$\begin{array}{l}\text { Fiber-premixed } \\
\text { indirect resin } \\
\text { composite }\end{array}$} & $\begin{array}{l}\text { Jacket Opaque JMO } \\
\text { (A3 body shade) }\end{array}$ & FMC & UDPAC, TEGDMA & $\begin{array}{l}\text { Sun Medical } \\
\text { Co. Ltd. }\end{array}$ & $\mathrm{RS} 3$ \\
\hline & & & $\begin{array}{l}\text { Silanized milled-glass fiber } \\
(10-\mu \mathrm{m} \text { diameter, } 150-\mu \mathrm{m} \text { length) } \\
\text { Colloidal silica }(0.007 \mu \mathrm{m}) \\
\text { Photoinitiator, Stabilizer }\end{array}$ & & \\
\hline \multirow[t]{2}{*}{$\begin{array}{l}\text { Indirect resin } \\
\text { composite }\end{array}$} & $\begin{array}{l}\text { Meta Color Prime } \\
\text { Art Body Paste A3- } \\
\text { B (A3 body paste) }\end{array}$ & IC & UDMA, TEGDMA & $\begin{array}{l}\text { Sun Medical } \\
\text { Co. Ltd. }\end{array}$ & $\mathrm{RS} 1$ \\
\hline & & & $\begin{array}{l}\text { Reactive prepolymerized filler } \\
\text { Photoinitiator }\end{array}$ & & \\
\hline \multirow[t]{4}{*}{$\begin{array}{l}\text { Luting } \\
\text { materials }\end{array}$} & Panavia F2.0 & PV & $\begin{array}{c}\text { Paste A: MDP, Methacrylate monomer, Filler, } \\
\text { Photoinitiator, Chemical initiator }\end{array}$ & $\begin{array}{l}\text { Kuraray } \\
\text { Medical Inc. }\end{array}$ & 00117A \\
\hline & & & $\begin{array}{l}\text { Paste B: Methacrylate monomer } \\
\text { Photoinitiator, Chemical initiator }\end{array}$ & & 00087A \\
\hline & & & Oxyguard II: Polyethylene glycol, Accelerator & & $00525 \mathrm{~A}$ \\
\hline & Super-Bond C\&B & SB & $\begin{array}{l}\text { Catalyst: TBB } \\
\text { Monomer Liquid: 4-META, MMA } \\
\text { Powder (Clear): PMMA }\end{array}$ & $\begin{array}{l}\text { Sun Medical } \\
\text { Co. Ltd. }\end{array}$ & $\begin{array}{l}\text { LV11 } \\
\text { LS2 } \\
\text { LV21 }\end{array}$ \\
\hline
\end{tabular}

UDPAC: urethane dimethacrylate with polyaliphatic carbonate segment

TEGDMA: triethylene glycol dimethacrylate

UDMA: urethane dimethacrylate

MDP: 10-methacryloyloxydecyl dihydrogen phosphate

TBB: tri- $n$-butylborane derivative

4-META: 4-methacryloxyethyl trimellitate anhydrate

MMA: methyl methacrylate

PMMA: poly(methyl methacrylate) 
technique. Impressions were poured with a type IV improved dental stone (Suprastone, Kerr Corp., Orange, CA, USA). After setting, the stone dies were removed from the impressions and coated with a proprietary separating medium (Prime Sep, Sun Medical Co. Ltd.) of $10 \mu \mathrm{m}$ thickness.

\section{Specimen fabrication}

Table 2 summarizes the characteristics of the fabricated specimens. Half of the specimens $(n=14)$ were fabricated without using the FMC, and the remaining specimens $(n=14)$ were fabricated with FMC.

The FMC substructures were layered in three increments into a final thickness of approximately 0.5 $\mathrm{mm}$. The thickness of each increment was measured using a customized periodontal probe which allowed 0.2-mm depth measurements, and each increment was polymerized in a laboratory photopolymerizing unit $(\alpha-$ Light II, Morita, Tokyo, Japan) for 90 seconds. Indirect resin composite was built up in two increments to acquire an inlay with simplified occlusal surface configuration. Each increment was polymerized in the laboratory photopolymerizing unit ( $\alpha$-Light II, Morita) for 90 seconds. All the inlays were finished with silicone polishing burs (CompoMaster, Shofu, Kyoto, Japan) and removed from the stone dies.

The inner surfaces of the inlays were air-abraded with $50-\mu \mathrm{m}$ aluminum oxide particles (Hi Aluminas, Shofu) under $0.2 \mathrm{MPa}$ for 5 seconds, and then thoroughly air-blasted. For both with-FMC and without-FMC groups, half of the inlay preparations $(n=7)$ were cemented into the prepared cavities using either SB or PV.

Prior to cementation, the enamel surfaces of prepared cavities were etched with $65 \%$ phosphoric acid (Red Activator, Sun Medical) for 30 seconds for SB-bonded groups, and 40\% phosphoric acid (K-etchant Gel, Kuraray Medical) for 10 seconds for PV-bonded groups. Following which, the dentin surfaces were treated with a dentin conditioner (Green Activator, Sun Medical) for 5 seconds for SB-bonded groups, and a dentin primer (ED Primer II, Kuraray Medical) for 30 seconds for PV-bonded groups. The inlays were cemented into the prepared cavities according to manufacturers' instructions under a $1.0 \mathrm{kgf}$ static load, and excess cement was removed. A total of 28 specimens, namely seven specimens for four groups, were thus fabricated (Fig. 3).
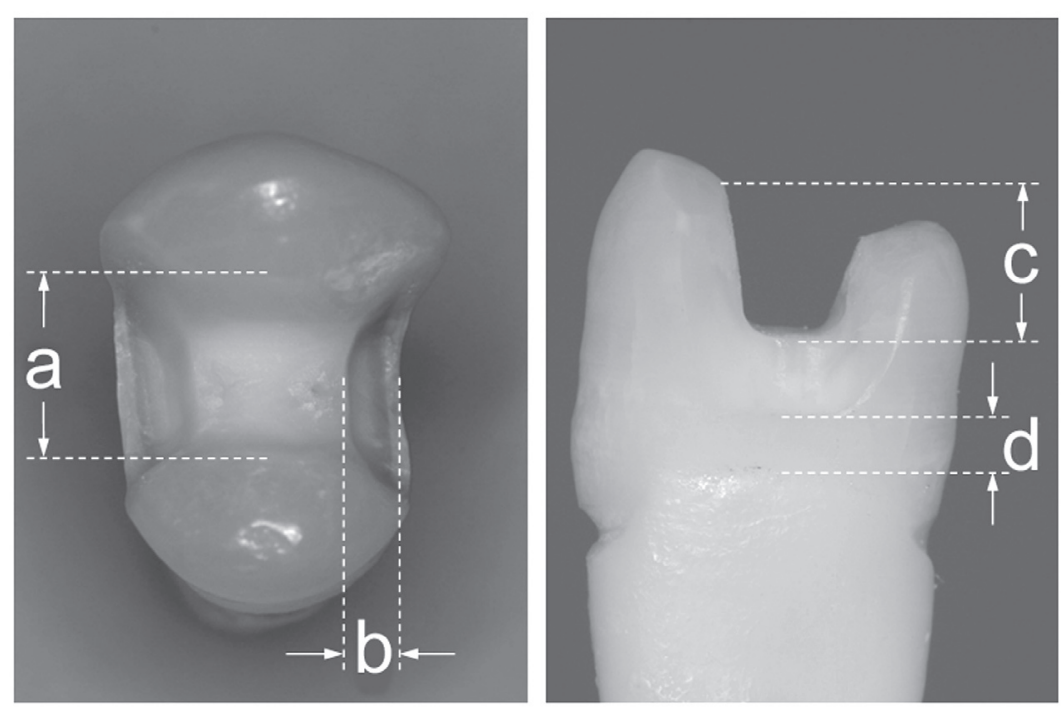

Fig. 2 Dimensions of inlay preparation:

a: $3.2 \mathrm{~mm}$ (same as the diameter of SF206CR bur)

b: $1.4 \mathrm{~mm}$ (same as the diameter of SF103CR bur)

c: $3.6 \mathrm{~mm}$ (same depth and taper of SF206CR bur)

$\mathrm{d}: 1.0 \mathrm{~mm}$ above the cement-enamel junction

Table 2 Characteristics of fabricated specimens in four different groups

\begin{tabular}{|c|c|c|}
\hline Group name & Inlay materials & Adhesive cements \\
\hline $\mathrm{IC} / \mathrm{SB}$ & Indirect resin composite & Super-Bond C\&B \\
\hline $\mathrm{FMC} / \mathrm{IC} / \mathrm{SB}$ & Fiber-premixed indirect resin composite/Indirect resin composite & Super-Bond C\&B \\
\hline $\mathrm{IC} / \mathrm{PV}$ & Indirect resin composite & Panavia F 2.0 \\
\hline $\mathrm{FMC} / \mathrm{IC} / \mathrm{PV}$ & Fiber-premixed indirect resin composite/Indirect resin composite & Panavia F 2.0 \\
\hline
\end{tabular}




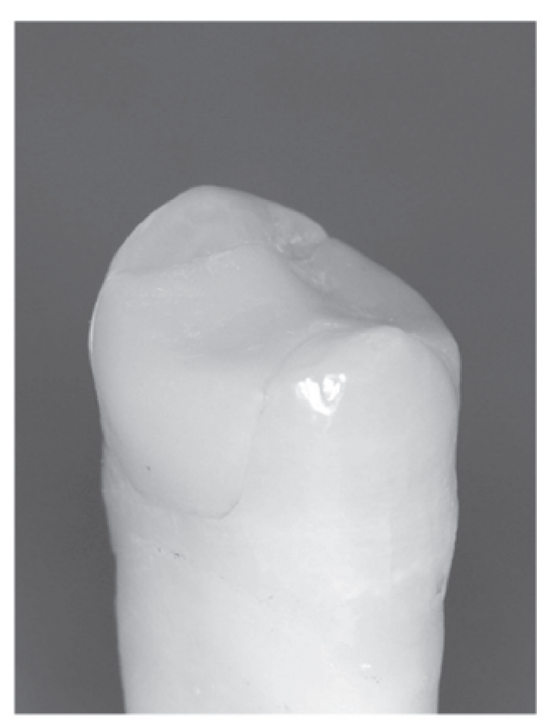

Fig. 3 MOD composite inlay cemented into the prepared cavity.

\section{Fracture test}

Specimens were removed from the molds and stored in $37^{\circ} \mathrm{C}$ distilled water for 24 hours. After water storage, the specimens were subjected to thermocycling between $4^{\circ} \mathrm{C}$ and $60^{\circ} \mathrm{C}$ with 1-minute dwell time at each temperature for 5,000 cycles. Each specimen was remounted into the mold, and a fracture test was carried out using a universal testing machine (Autograph AGS-5kNG, Shimadzu Corp., Kyoto, Japan). A vertical load was applied through a 4-mmdiameter sphere onto the mesial occlusal pit of the composite inlay at a crosshead speed of $1.0 \mathrm{~mm} / \mathrm{min}$ until initial crack occurred.

Load-displacement curve was recorded throughout the fracture test using an analog recorder (AR-6422, Shimadzu Corp.) at a chart speed of $100 \mathrm{~mm} / \mathrm{min}$. The load at initial crack was determined as failure load (N). The initial crack was detected as the first sharp drop in the load (Fig. 4) with a snapping sound. The energy required to cause initial crack $(J)$ was calculated from the area under the load-displacement curve. All the load-displacement curves were captured using a scanner (GT-9800F, Seiko Epson Corp., Nagano, Japan). To calculate failure energy (J), the area bounded by the load-displacement curve and the $\mathrm{x}$-axis until the initial crack occurred was computed using an image analysis software (Microanalyzer, Nihon Poladigital Corp., Tokyo, Japan).

\section{Statistical analysis}

Both failure load and failure energy data of each group were analyzed and depicted with box and whisker plots using a statistical analysis software (SPSS 13.0, SPSS Inc., Chicago, IL, USA). Each box represented the 25th

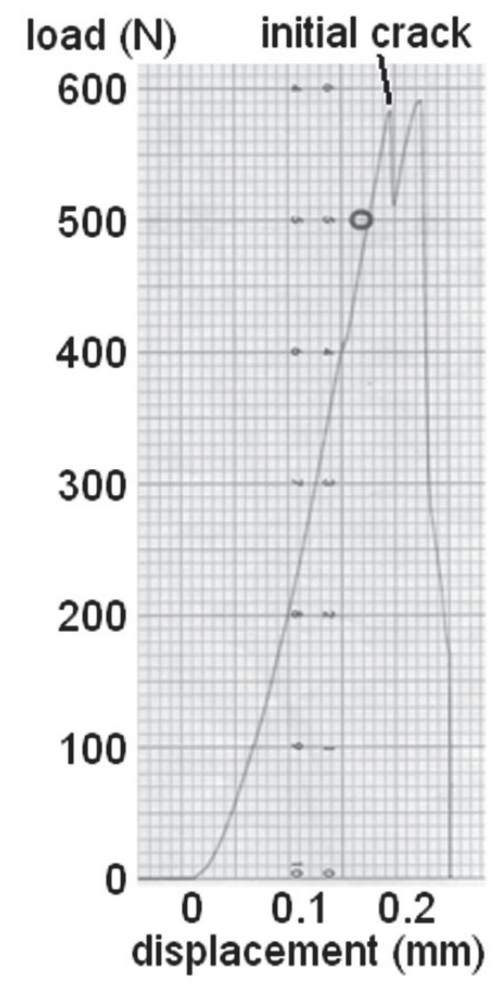

Fig. 4 Typical trace of a load-displacement curve during fracture test.

and 75th percentiles, and the bar represented the median. Whiskers were drawn to show the range 1.5 times the interquartile range beyond the 25 th and 75 th percentiles. For values outside 1.5 times the interquartile range, they were marked as outliers ("o").

Owing to large variations in both the failure load and failure energy data, non-parametric KruskalWallis test was performed to determine significant differences among the four groups. The Mann-Whitney $\mathrm{U}$ test was used for subsequent comparisons between groups. The level of significance was set at 0.05 for all statistical analyses.

\section{RESULTS}

Figures 5 and 6 present the failure load $(\mathrm{N})$ and failure energy $(J)$ results for each group.

On failure load, Kruskal-Wallis test showed that there were no significant differences among the four groups ( $p=0.1081$ ) (Fig. 5).

On failure energy, Kruskal-Wallis test showed that there were significant differences among the four groups ( $p=0.0034$ ) (Fig. 6). The Mann-Whitney U test showed that the mean failure energy of $\mathrm{FMC/IC/SB}$ group was significantly higher than the other three groups $(p=0.004-0.018)$. Between the SB-bonded groups, the failure energy of FMC/IC/SB group was significantly higher than that of IC/SB group 


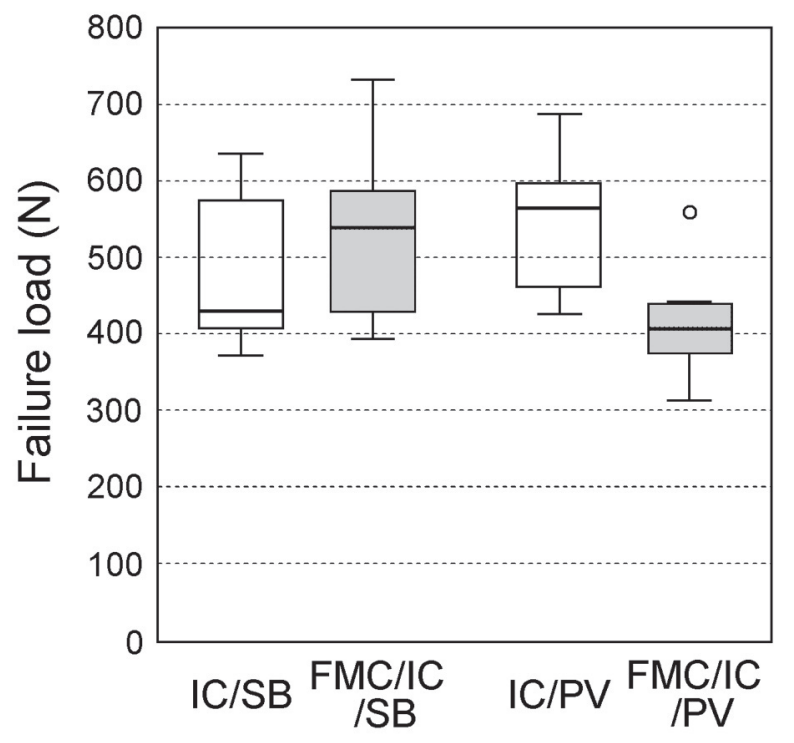

Fig. 5 Failure load of each group.

$(p=0.0181)$. In other words, the fiber substructure exerted a significant positive effect on the SB-bonded specimens. Between the PV-bonded groups, failure energy of $\mathrm{FMC/IC/PV}$ group was significantly lower than that of IC/PV group $(p=0.035)$. In other words, the fiber substructure exerted a negative effect on the PV-bonded specimens.

Initial cracks were observed in the composite resin inlays, and fracture of tooth structure did not occur for all groups. No delamination of FMC from IC was found for the with-FMC groups.

\section{DISCUSSION}

This in vitro study evaluated the effect of fiberpremixed indirect resin composite, as a substructure, on the fracture resistance of class II indirect composite restorations adhered with two different adhesive cements under static loading. The hypothesis was partially accepted because the fiber substructure showed a significant positive effect on the failure energy of SB-bonded specimens, but there was no significant effect on failure strength.

A previous study ${ }^{12}$ reported that the impact resistance of indirect composite was affected by the thickness of FMC. Therefore, in this study, influence of this variable was stringently excluded by measuring each element of the cavity preparation to ensure uniform FMC thickness.

The maximum bite force and single tooth bite force in the premolar region have been reported to be 246$398 \mathrm{~N}^{13)}$ and 179-291 $\mathrm{N}^{14)}$ respectively. The failure loads obtained for all the groups in this study were greater than these values. In particular, the failure loads obtained in this study agreed with the mean

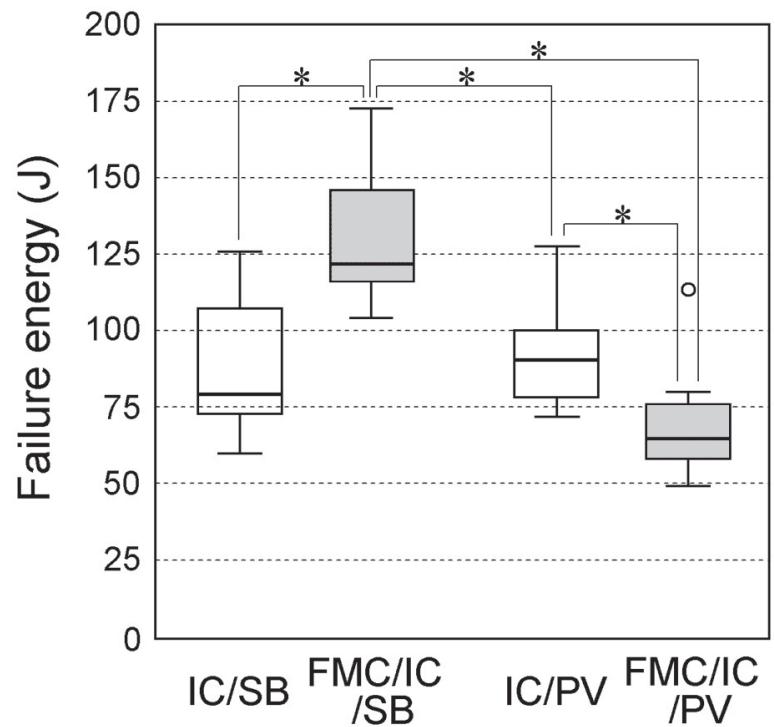

Fig. 6 Failure energy of each group. The asterisks denote significant difference $(p<0.05)$.

value of $422 \mathrm{~N}$ as reported by Sorrentino et al. ${ }^{15)}$. Notably, loading in the study of Sorrentino et al. ${ }^{15)}$ was performed in a similar manner as the current study. Therefore, the specimen preparation conditions employed in this study might be acceptable for clinical simulation.

The UDPAC monomer used for FMC possesses a soft segment of polyaliphatic carbonate segment ${ }^{12)}$. While the high impact resistance of FMC could be attributed chiefly to the silanized milled glass fiber (Table 1), the ductile property of the FMC should be ascribed to the soft segment of the UDPAC monomer. Indeed, the ductile property of FMC seemed to be more pronounced when compared against the stiff properties of indirect resin composite, as the fracture energy of the FMC was approximately 3.5 times that of indirect resin composite ${ }^{12)}$. Nonetheless, combining these two different types of materials might have improved the impact resistance of the indirect rein composite, as gleaned from the results of the present study.

For the FMC/IC/SB group, the intermediate layer consisting of FMC and SB might have functioned as a shock-absorbing structure between the composite resin and dentin. Consequently, a significantly higher failure energy was obtained for $\mathrm{FMC} / \mathrm{IC} / \mathrm{SB}$ group as compared to group IC/SB. The clinical implication of this finding could be a prolonged life of composite resin inlays in intra-oral conditions. In sharp contrast, the failure energy of PV-bonded specimens declined when combined with FMC. It should be pointed out that for efficacious reinforcement of indirect resin composites, the FMC substructure must be approximately $30 \%$ in thickness ${ }^{12)}$. However, the stiff PV layer between FMC and dentin in the FMC/IC/PV group reduced the relative thickness of FMC within the total thickness of 
the inlay-PV complex. Consequently, this phenomenon blunted the effectiveness of FMC as a shock absorber, thereby resulting in significantly lower failure energy of group FMC/IC/PV as compared to groups IC/PV and FMC/IC/SB.

To date, many studies have reported on the dentin bond strengths of the adhesive resin cements tested in this study ${ }^{16-19)}$. In the study by Al-Assaf et al. ${ }^{16)}$, it was reported that PV showed comparable bond strength with SB. On the other hand, other studies ${ }^{17-19)}$ reported that SB exhibited higher bond strength than PV. As for the bonding between the adhesive resin cements and restorative materials employed in this study, Taira et al. reported that the FMC showed superior bond strength compared to indirect resin composite ${ }^{20)}$. As for the comparison between SB and PV, SB showed significantly higher bond strength when bonded to indirect resin composite but no significant differences were observed for the bonding to $\mathrm{FMC}^{20)}$. In the present study, there were no significant differences in failure load among the four groups. Against this backdrop of contradictory and confusing results, the clinical significance of FMC toward the fracture resistance of restored premolars needs to be further evaluated to ensure the longevity of restorations and restored teeth.

\section{CONCLUSION}

Within the limitations of this in vitro study and based on the results obtained, it was shown that the use of fiber-premixed indirect resin composite (Jacket Opaque) for indirect composite (Meta Color Prime Art) MOD inlays enhanced the fracture energy when cemented to dentin using Super-Bond C\&B.

\section{REFERENCES}

1) Da Fonte Porto Carreiro A, Dos Santos Cruz CA, Vergani CE. Hardness and compressive strength of indirect composite resins: effects of immersion in distilled water. $\mathrm{J}$ Oral Rehabil 2004; 31: 1085-1089.

2) Suzuki S, Nagai E, Taira Y, Minesaki Y. In vitro wear of indirect composite restoratives. J Prosthet Dent 2002; 88: 431-436.

3) Nakamura T, Saito O, Mizuno M, Tanaka H. Changes in translucency and color of particulate filler composite resins. Int J Prosthodont 2002; 15: 494-499.

4) de Menezes ML, Arrais CA, Giannini M. Influence of lightactivated and auto- and dual- polymerizing adhesive systems on bond strength of indirect composite resin to dentin. J Prosthet Dent 2006; 96: 115-121.
5) Lehmann F, Eickemeyer G, Rammelsberg P. Fracture resistance of metal-free composite crowns - effects of fiber reiforcement, thermal cycling, and cementation technique. J Prosthet Dent 2004; 92: 258-264.

6) Ohlmann B, Dreyhaupt J, Schmitter M, Gabbert O, Hassel A, Rammelsberg P. Clinical performance of posterior metalfree polymer crowns with and without fiber reinforcement: one-year results of a randomized clinical trial. J Dent 2006; 34: 757-762.

7) Waki T, Nakamura T, Nakamura T, Kinuta S, Wakabayashi K, Yatani H. Fracture resistance of inlay-retained fixed partial dentures reinforced with fiber-reinforced composite. Dent Mater J 2006; 25: 1-6.

8) Stiesch-Scholz M, Schulz K, Borchers L. In vitro fracture resistance of four-unit fiber-reinforced composite fixed partial dentures. Dent Mater 2006; 22: 374-381.

9) Behr M, Rosentritt M, Latzel D, Handel G. Fracture resistance of fiber-reinforced vs. non-fiber-reinforced composite molar crowns. Clin Oral Invest 2003; 7: 135-139.

10) Ellakwa A, Thomas GD, Shortall AC, Marquis PM, Burke FJ. Fracture resistance of fiber-reinforced composite crown restorations. Am J Dent 2003; 16: 375-380.

11) Suzuki S, Ori T, Saimi Y. Effects of filler composition on flexibility of microfilled resin composite. J Biomed Mater Res B Appl Biomater 2005; 74: 547-552.

12) Suzuki S, Saimi Y, Ono T. Evaluation of a new fiberreinforced resin composite. J Biomed Mater Res B Appl Biomater 2006; 76: 184-189.

13) Fontijn-Tekamp FA, Slagter AP, van der Bilt A, van’T Hof MA, Witter DJ, Kalk W, Jansen JA. Biting and chewing in overdentures, full dentures, and natural dentitions. J Dent Res 2000; 79: 1519-1524.

14) Ferrario VF, Sforza C, Serrao G, Dellavia C, Tartaglia GM. Single tooth bite forces in healthy young adults. J Oral Rehabil 2004; 31: 18-22.

15) Sorrentino R, Monticelli F, Goracci C, Zarone F, Tay FR, Garcia-Godoy F, Ferrari M. Effect of post-retained composite restorations and amount of coronal residual structure on the fracture resistance of endodontically-treated teeth. Am J Dent 2007; 20: 269-274.

16) Al-Assaf K, Chakmakchi M, Palaghias G, Karanika-Kouma A, Eliades G. Interfacial characteristics of adhesive luting resins and composites with dentine. Dent Mater 2007; 23: 829-839.

17) Mak YF, Lai SC, Cheung GS, Chan AW, Tay FR, Pashley DH. Micro-tensile bond testing of resin cements to dentin and an indirect resin composite. Dent Mater 2002; 18: 609621.

18) Soeno K, Suzuki S, Yokomichi R, Taira Y, Atsuta M. Evaluation of a novel dentin bonding system compared to commercial bonding system. J Dent 2004; 32: 315-320.

19) Yang B, Ludwig K, Adelung R, Kern M. Micro-tensile bond strength of three luting resins to human regional dentin. Dent Mater 2006; 22: 45-56.

20) Taira Y, Sakai M, Yang L, Sawase T, Atsuta M. Bond strength between luting materials and a fiber-reinforced resin composite for indirect restorations. Dent Mater J 2007; 26: 628-634. 